\title{
Wave fronts may move upstream in semiconductor superlattices
}

\author{
A. Carpio \\ Departamento de Matemática Aplicada, Universidad Complutense, Madrid 28040, Spain \\ L. L. Bonilla \\ Departamento de Matemáticas, Universidad Carlos III de Madrid, Avenida de la Universidad 30, 28911 Leganés, Spain \\ A. Wacker and E. Schöll \\ Institut für Theoretische Physik, Technische Universität Berlin, Hardenbergstrasse 36, 10623 Berlin, Germany
}

(Received 29 November 1999)

\begin{abstract}
In weakly coupled, current biased, doped semiconductor superlattices, domain walls may move upstream against the flow of electrons. For appropriate doping values, a domain wall separating two electric-field domains moves downstream below a first critical current, it remains stationary between this value and a second critical current, and then moves upstream above. These conclusions are reached by using a comparison principle to analyze a discrete drift-diffusion model, and validated by numerical simulations. Possible experimental realizations are suggested.

PACS number(s): 05.45.-a, 72.20.Ht, 73.61.- $-\mathrm{r}$
\end{abstract}

\section{INTRODUCTION}

Current instabilities in doped semiconductor superlattices (SL's) have been an active subject of research during this decade. For strongly coupled SL's, Bloch oscillations [1-3] and Wannier-Stark hopping [4] produce negative differential conductivity at high electric fields. This may result in selfsustained oscillations of the current due to recycling of charge dipole domains as in the Gunn effect of bulk $n$-type GaAs [5,2]. For weakly coupled SL's, sequential tunneling is the main mechanism of vertical transport. Under de voltage bias conditions, stationary electric field domains may form if doping is large enough [6,7]. Below a critical doping value, the existing charge inside the SL may not be able to pin domain walls, and current self-oscillations appear [8,9]. These oscillations may be due to recycling of charge monopoles (domain walls) or dipoles depending on the boundary condition at the injecting contact region (in a typical $n^{+}-n-n^{+}$configuration with the SL imbedded between highly doped regions, the doping at the emitter region is crucial) [10]. Driven chaotic oscillations have also been predicted [11] and observed in experiments [12]. Finally, there are ways to tune the charge inside the SL (and therefore obtain stationary domains or self-oscillations) without replacing it by a different one, for example, by applying a transverse magnetic field [13] or by photoexciting the SL [14].

Transport in weakly coupled SL's can be described by simple rate equation models for electron densities and average fields in the wells, [15-18]. Many of the effects related above have been explained by means of a simple discrete drift model $[16,17,19,20]$. In this model, the tunneling current between two adjacent wells, $J_{i \rightarrow i+1}$, equals the twodimensional (2D) electron charge density at well $i$ times a drift velocity, which depends on the electric field at the same well. By starting from a microscopic sequential tunneling model, it has been shown that the discrete drift model is a good approximation at low temperatures and for fields above the first plateau of the SL current-voltage characteristic $[18,21]$. For low dc voltages on the first plateau, a discrete diffusion (which is a nonlinear function of the field) should be added. This term contains the contribution to $J_{i \rightarrow i+1}$ of the tunneling from well $i+1$ back to well $i$ (which vanishes for large enough electric fields) [18,21]. In this paper we report an interesting consequence of electron diffusivity at low fields: if the current is sufficiently high, and so is the doping, a domain wall (monopole wave) which connects two domains may travel in a direction opposite to the flow direction for electrons (i.e., upstream, in the positive current direction). This striking phenomenon is contrary to the usual situation: a monopole either moves downstream (in the direction of the flow of electrons), or it remains stationary, [19]. We substantiate our claim both by numerical simulations of the discrete drift-diffusion model and by rigorous mathematical analysis based upon a comparison principle [22]. Mathematical analysis yields useful bounds for critical values of current and well doping, and for monopole velocity.

There are related fields for which differential-difference equations (similar to discrete drift-diffusion models) model the systems of interest. These include propagation of nerve impulses along myelinated fibers, modeled by discrete FitzHugh-Nagumo equations [23,24]; motion of dislocations $[25,26]$ and sliding charge density waves [27], modeled by variants of the Frenkel-Kontorova model [28]; etc. The theory of wave front propagation was developed for some of these models, which are simpler than ours: convection is typically absent from them and diffusion is purely linear [24].

The rest of the paper is as follows. We write the driftdiffusion model with appropriate boundary conditions in Sec. II. There we render these equations dimensionless, and explain the results of numerical simulations on a current biased infinitely long SL's. Furthermore, we find by numerical simulations that our results for infinite SL's may be realized in finite SL's with appropriate boundary conditions under 

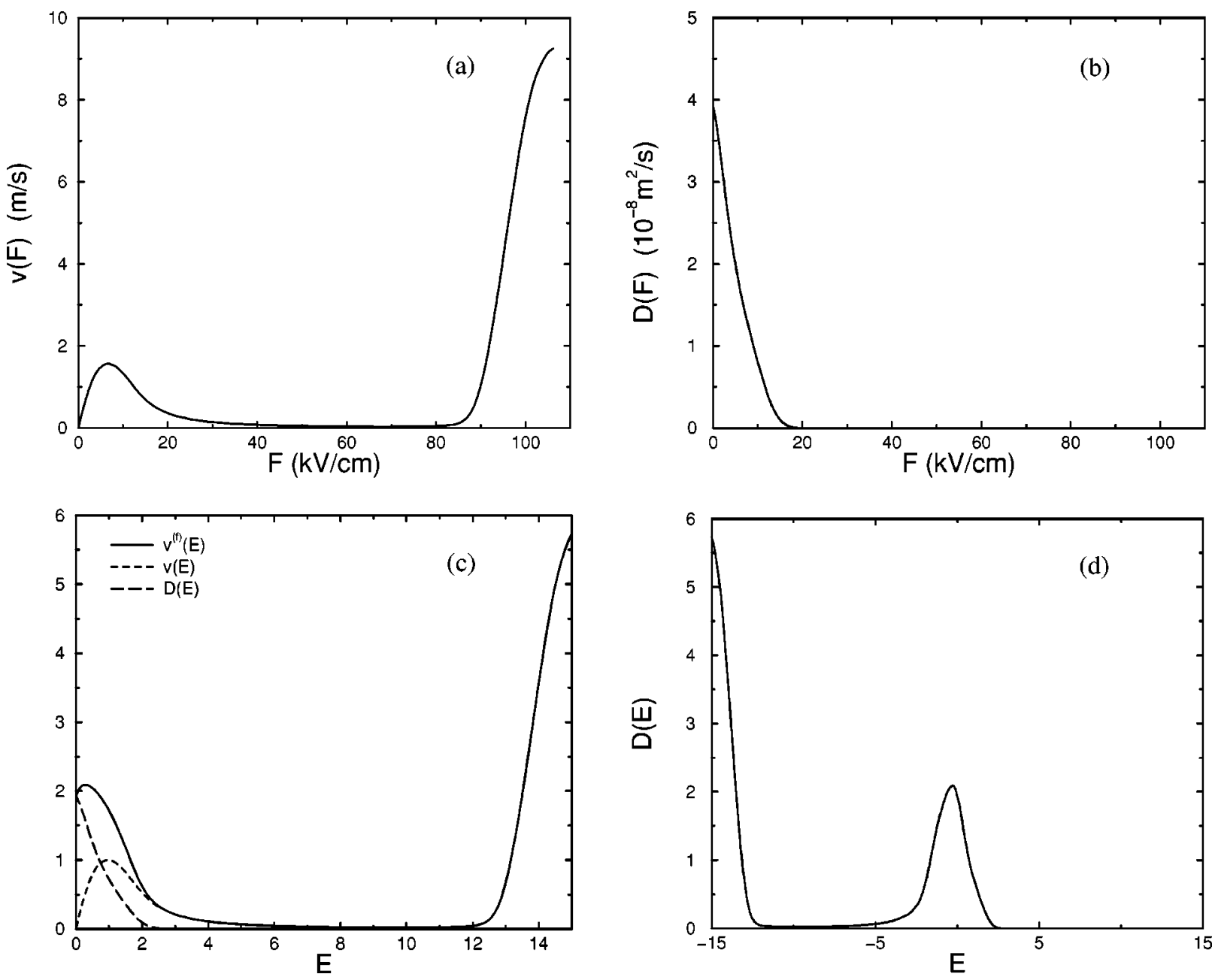

FIG. 1. (a) Drift velocity and (b) diffusion coefficient for a 9-nm GaAs/4-nm AlAs SL. Doping at the wells is $N_{D}^{w}=1.5 \times 10^{11} \mathrm{~cm}^{-2}$, whereas at the contact regions, $N_{D}=2 \times 10^{18} \mathrm{~cm}^{-3}$. (c) Dimensionless drift velocity $v(E)$, diffusion (equivalent to backward tunneling velocity) $D(E)$, and forward tunneling velocity $v^{(f)}(E)=v(E)+D(E)$. (d) Extension of the dimensionless diffusivity to negative values of field. We have $D(-E)=v^{(f)}(E)$. The same formula yields the extension of $v^{(f)}(E)$ to negative fields. Then $v(E)$ is an odd function of $E$.

constant current bias. A theoretical analysis based on the comparison principle is presented in Sec. III. Section IV contains our conclusions. Finally some material of a more technical nature is relegated to the Appendixes.

\section{DISCRETE DRIFT-DIFFUSION MODEL}

\section{A. Equations and boundary conditions}

At low enough temperatures (much less than a typical Fermi energy of a SL well measured from the first subband, say $20 \mathrm{meV}$ or $232 \mathrm{~K}$ ), the following discrete drift-diffusion equations model sequential vertical transport in a weakly doped SL $[18,21]$ :

$$
\begin{gathered}
\frac{\varepsilon}{e} \frac{d F_{i}}{d t}+\frac{n_{i} v\left(F_{i}\right)}{d+w}-D\left(F_{i}\right) \frac{n_{i+1}-n_{i}}{(d+w)^{2}}=J(t), \\
F_{i}-F_{i-1}=\frac{e}{\varepsilon}\left(n_{i}-N_{D}^{w}\right) .
\end{gathered}
$$

Equation (1) is Ampère's law establishig that the total current density $e J$ is the sum of displacement and tunneling currents. The latter consists of a drift term, $e n_{i} v\left(F_{i}\right) /(d$ $+w)$, and a diffusion term, $e D\left(F_{i}\right)\left(n_{i+1}-n_{i}\right) /(d+w)^{2}$. We have adopted the convention (typical in this field) that the current density has the same direction as the flow of electrons. Equation (1) holds for $i=1, \ldots, N-1$. Equation (2) is the Poisson equation, and it holds for $i=1, \ldots, N . n_{i}$ is the $2 \mathrm{D}$ electron number density at well $i$, which is singularly concentrated on a plane located at the end of the well. $F_{i}$ is minus an average electric field on a SL period comprising the $i$ th well and the $i$ th barrier (well $i$ lies between barriers $i$ -1 and $i$; barriers 0 and $N$ separate the SL from the emitter and collector contact regions, respectively). Parameters $\varepsilon, d$, $w$, and $N_{D}^{w}$ are well permittivity, barrier width, well width, and $2 \mathrm{D}$ doping in the wells, respectively.

Drift velocity and diffusion coefficient are depicted in Fig. 1 for the 9-nmGaAs/4-nmAlAs SL of Ref. [9]. We have obtained them from microscopic calculations presented in Ref. [18] (which is appropriate for these sample parameters [29]) by setting $v(F)=J\left(N_{D}^{w}, N_{D}^{w}, F\right)(d+w) / N_{D}^{w}$ and $D(F)$ $=-\left[\partial J\left(N_{D}^{w}, N_{D}^{w}, F\right) / \partial n_{i+1}\right](d+w)^{2}$. Here $e J\left(n_{i}, n_{i+1}, F_{i}\right)$ is the tunneling current between wells $i$ and $i+1, J_{i \rightarrow i+1}$. We assume that the tunneling current is a function of the average field at the $i$ th SL period, $F_{i}=F$, and of the 2D electron densities at wells $i$ and $i+1, n_{i}$ and $n_{i+1}$, respectively. Notice that our model for the tunneling current, 


$$
\begin{aligned}
e J\left(n_{i}, n_{i+1}, F_{i}\right) & =\frac{e n_{i} v\left(F_{i}\right)}{d+w}-e D\left(F_{i}\right) \frac{n_{i+1}-n_{i}}{(d+w)^{2}} \\
& \equiv \frac{e n_{i} v^{(f)}\left(F_{i}\right)-e n_{i+1} v^{(b)}\left(F_{i}\right)}{d+w}
\end{aligned}
$$

is reasonable for temperatures much lower than a typical Fermi energy in the wells measured from the first subband (say $20 \mathrm{meV}$ ), [21]. The tunneling current density should change sign if we reverse the electric field and exchange the electron densities at wells $i$ and $i+1$ : $J\left(n_{i}, n_{i+1}, F_{i}\right)$ $=-J\left(n_{i+1}, n_{i},-F_{i}\right)$. This inversion symmetry implies

$$
v^{(f)}(-F)=v^{(b)}(F) \quad \text { and } \quad v(-F)=-v(F),
$$

where $\quad v^{(b)}(F)=D(F) /(d+w) \quad$ and $\quad v^{(f)}(F)=v(F)$ $+v^{(b)}(F)$. See Fig. 1(d).

Equations (1) and (2) should be supplemented with appropriate bias, initial, and boundary conditions. Among possible bias conditions, we shall consider the extreme cases of current bias $[J(t)$ specified $]$ and voltage bias

$$
(d+w) \sum_{i=1}^{N} F_{i}=V,
$$

with specified $V=V(t)$. Using Eq. (4) ignores potential drops at the contact regions and at barrier 0 , and it overestimates the contribution of barrier $N$ by a factor $1+w / d$ [21]. These contributions are negligible for long SL's $(N=40$ or larger), so that we shall adopt the simpler expression (4). Appropriate boundary conditions have been derived under the same approximations as in Eq. (1) [21]. They are

$$
\begin{gathered}
\frac{\varepsilon}{e} \frac{d F_{0}}{d t}+j_{e}^{(f)}\left(F_{0}\right)-\frac{n_{1} w^{(b)}\left(F_{0}\right)}{d+w}=J(t), \\
\frac{\varepsilon}{e} \frac{d F_{N}}{d t}+\frac{n_{N} w^{(f)}\left(F_{N}\right)}{d+w}=J(t),
\end{gathered}
$$

where the emitter current density $e j_{e}^{(f)}(F)$, the emitter backward velocity $w^{(b)}(F)$, and the collector forward velocity $w^{(f)}(F)$ are functions of the electric field depicted in Fig. 3 of Ref. [21] for contact regions similar to those used in experiments [9].

To analyze the discrete drift-diffusion model, it is convenient to render all equations dimensionless. Let $v(F)$ reach its first positive maximum at $\left(F_{M}, v_{M}\right)$. We adopt $F_{M}$, $N_{D}^{w}, v_{M}, v_{M}(d+w), e N_{D}^{w} v_{M} /(d+w)$, and $\varepsilon F_{M}(d+w) /$ $\left(e N_{D}^{w} v_{M}\right)$ as the units of $F_{i}, n_{i}, v(F), D(F), e J$, and $t$, respectively. For the first plateau of the 9/4 SL of Ref. [9], we find $F_{M}=6.92 \mathrm{kV} / \mathrm{cm}, N_{D}^{w}=1.5 \times 10^{11} \mathrm{~cm}^{-2}, v_{M}=156$ $\mathrm{cm} / \mathrm{s}, v_{M}(d+w)=2.03 \times 10^{-4} \mathrm{~cm}^{2} / \mathrm{s}$, and $e N_{D}^{w} v_{M} /(d+w)$ $=2.88 \mathrm{~A} / \mathrm{cm}^{2}$. The units of current and time are $0.326 \mathrm{~mA}$ and $2.76 \mathrm{~ns}$, respectively. Then Eqs. (1)-(4) become

$$
\begin{gathered}
\frac{d E_{i}}{d t}+v\left(E_{i}\right) n_{i}-D\left(E_{i}\right)\left(n_{i+1}-n_{i}\right)=J, \\
E_{i}-E_{i-1}=\nu\left(n_{i}-1\right),
\end{gathered}
$$

$$
\frac{1}{N} \sum_{i=1}^{N} E_{i}=\phi
$$

Here we have used the same symbols for dimensional and dimensionless quantities except for the electric field ( $F$ dimensional, $E$ dimensionless). The parameters $\nu=e N_{D}^{w} /$ $\left(\varepsilon F_{M}\right)$ and $\phi=V /\left[F_{M} N(d+w)\right]$ are the dimensionless doping and average electric field (bias), respectively. For the 9/4 $\mathrm{SL}, \nu \approx 3$. We recall that $i=1, \ldots, N-1$ in Eq. (7) and $i$ $=1, \ldots, N$ in Eq. (8). The boundary conditions (5) and (6) become

$$
\frac{d E_{0}}{d t}+J_{e}\left(E_{0}\right)-w_{e}\left(E_{0}\right) n_{1}=J
$$

$$
\frac{d E_{N}}{d t}+w_{c}\left(E_{N}\right) n_{N}=J,
$$

where

$$
\begin{gathered}
J_{e}\left(E_{0}\right)=\frac{j_{e}^{(f)}\left(F_{M} E_{0}\right)(d+w)}{N_{D}^{w} v_{M}}, \\
w_{e}\left(E_{0}\right)=\frac{w^{(b)}\left(F_{M} E_{0}\right)}{v_{M}}, \\
w_{c}\left(E_{N}\right)=\frac{w^{(f)}\left(F_{M} E_{N}\right)}{v_{M}} .
\end{gathered}
$$

Figure 2 shows $J_{e}, w_{e}$, and $w_{c}$ as functions of the electric field. They are dimensionless versions of the curves plotted in Fig. 3 of Ref. [21].

\section{B. Numerical simulations}

Simple solutions of the drift-diffusion equations (7) and (8) under constant current bias are stationary or moving monopole wave fronts connecting two electric field domains. Let us consider monopole solutions with profiles $\left\{E_{i}\right\}$, which are increasing functions of $i$, for they are compatible with realistic boundary conditions in which the emitter region is highly doped [9]. We have simulated numerically on a large SL,

$$
\begin{aligned}
\frac{d E_{i}}{d t} & -\frac{D\left(E_{i}\right)+v\left(E_{i}\right)}{\nu}\left(E_{i-1}-E_{i}\right)-\frac{D\left(E_{i}\right)}{\nu}\left(E_{i+1}-E_{i}\right) \\
& =J-v\left(E_{i}\right)
\end{aligned}
$$

with fixed $J$, which is equivalent to Eqs. (7) and (8). Let $E^{(1)}(J)<E^{(2)}(J)<E^{(3)}(J)$ be the three solutions of $v(E)$ $=J$ for $v_{m}<J<1$, where $\left(E_{m}, v_{m}\right)$ is the minimum of $v(E)$ for $E>1$. For the $9 / 4 \mathrm{SL}$ of Fig. $1, E_{m}=9.8571$ and $v_{m}$ $=0.02192$. We have simulated Eq. (13) for different values of $\nu>0$ and of $J \in\left(v_{m}, 1\right)$. The initial condition was chosen so that $E_{i} \rightarrow E^{(1)}(J)$ as $i \rightarrow-\infty$, and $E_{i} \rightarrow E^{(3)}(J)$ as $i \rightarrow \infty$. We observed that, after a short transient, a variety of initial conditions sharing these features evolved toward either a stationary or moving monopole. For systematic numerical studies, we therefore adopted an initial steplike profile, with $E_{i}$ 

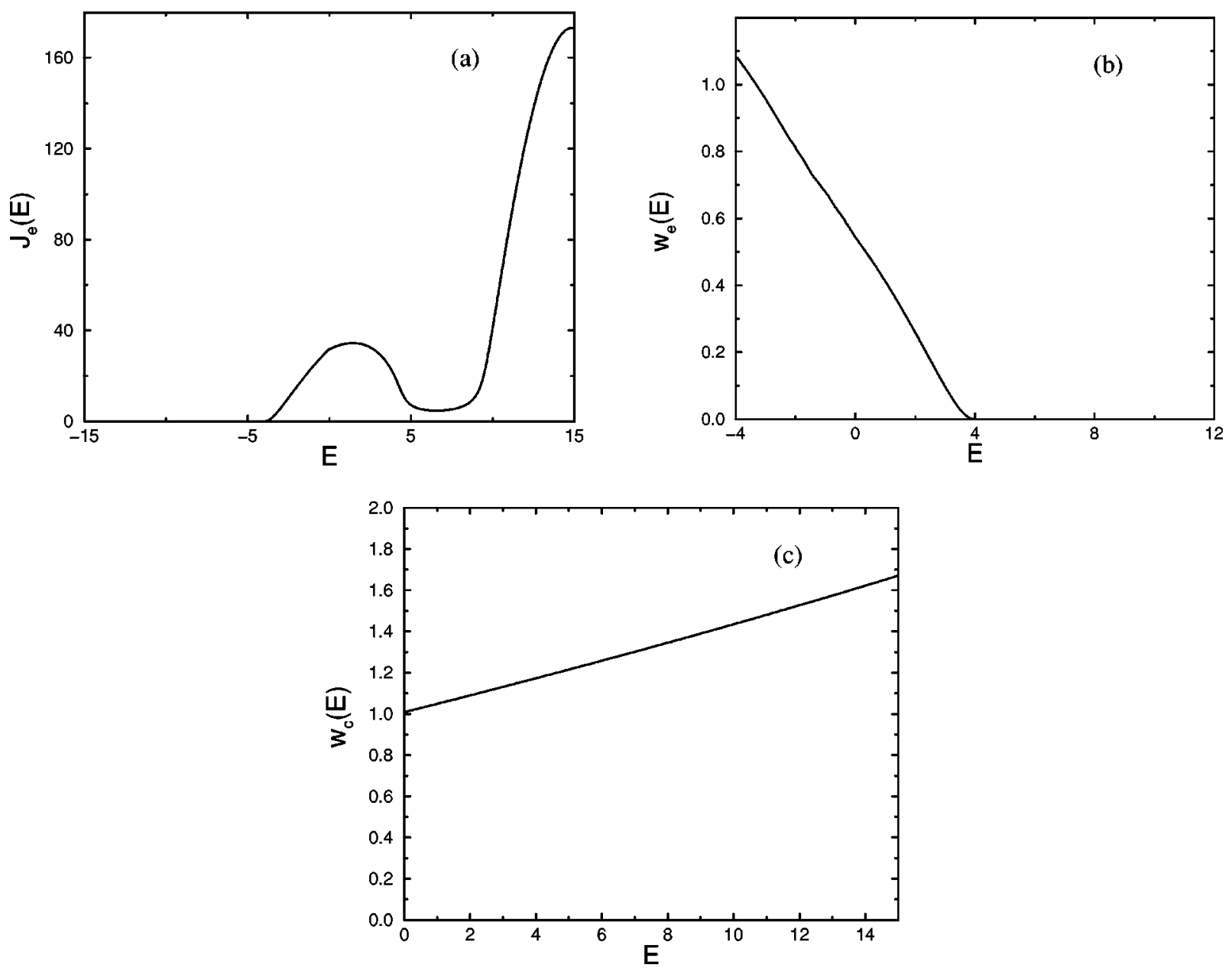

FIG. 2. Dimensionless functions of the electric field for the contact regions. (a) Current at the emitter, $J_{e}(E)$. (b) Backward velocity at the emitter, $w_{e}(E)$. (c) Forward velocity at the collector, $w_{c}(E)$.

$=E^{(1)}(J)$ for $i<0, E_{i}=E^{(3)}(J)$ for $i>0$ and $E_{0}=E^{(2)}(J)$. The boundary data were taken to be $E_{-N}=E^{(1)}(J), E_{N}$ $=E^{(3)}(J)$ with $N$ large.

Our results show that the dimensionless doping $\nu$ determines the type of solution of Eq. (13) which is stable. There are two important values of $\nu, \nu_{1}<\nu_{2}$.

(i) For $0<\nu<\nu_{1}$ and each fixed $J \in\left(v_{m}, 1\right)$, only traveling monopole fronts moving downstream (to the right) were observed. For $\nu>\nu_{1}$, stationary monopoles were found. According to the arguments of Wacker et al. [19] for the discrete drift model with $D(E)=0$, stationary monopoles exist for dimensionless doping larger than a critical value. An upper bound for this critical doping is

$$
\nu_{c}=v_{m} \frac{E_{m}-1}{1-v_{m}},
$$

which equals $\nu_{c}=0.198$ for our numerical example. We have found that $\nu_{1}=0.16$. This agreement with results obtained assuming $D(E)=0$ is not surprising: we shall prove in Sec. III that Eq. (14) holds as well for the model of Eqs. (7) and (8) with nonzero diffusivity.

(ii) For $\nu_{1}<\nu<\nu_{2}$, traveling fronts moving downstream exist only if $J \in\left[v_{m}, J_{1}(\nu)\right]$, where $J_{1}(\nu)<1$ is a critical value of the current. If $J \in\left[J_{1}(\nu), 1\right]$, the stable solutions are steady fronts (stationary monopoles). We have found that $\nu_{2}=0.33$.

(iii) New solutions are observed for $\nu>\nu_{2}$. As before, there are traveling fronts moving downstream if $J$ $\in\left[v_{m}, J_{1}(\nu)\right]$, and stationary monopoles if $J$ $\in\left[J_{1}(\nu), J_{2}(\nu)\right], J_{2}(\nu)<1$ is a new critical current. For $J_{2}(\nu)<J<1$, the stable solutions of Eq. (13) are monopoles traveling upstream (to the left). As $\nu$ increases, $J_{1}(\nu)$ and $J_{2}(\nu)$ approach $v_{m}$ and 1 , respectively. Thus stationary solutions are found for most values of $J$ if $\nu$ is large enough.

Figure 3 depicts $J_{1}(\nu)$ and $J_{2}(\nu)$ as functions of $\nu$. Notice that $J_{1}$ decreases from $J_{1}=1$ to $J_{1}=v_{m}$ as $\nu$ increases from $\nu_{1}$. Similarly, $J_{2}$ decreases from $J_{2}=1$ to a minimum value $J_{2} \approx 0.53$, and then increases back to $J_{2}=1$ as $\nu$ increases. The monopole velocity as a function of current is depicted in Fig. 4 for four different doping values, $\nu=0.5,1$, 3 , and 10 . For larger $\nu$, the interval of $J$ for which stationary solutions exist becomes wider again, trying to span the whole interval $\left(v_{m}, 1\right)$ as $\nu \rightarrow \infty$. For very large $\nu$, the velocities of downstream and upstream moving monopoles become extremely small in absolute value.

Notice that if we use the complete sequential tunneling current instead of the drift-diffusion approximation (3), in Eq. (1) the situation is the same. Figure 5 depicts monopole 


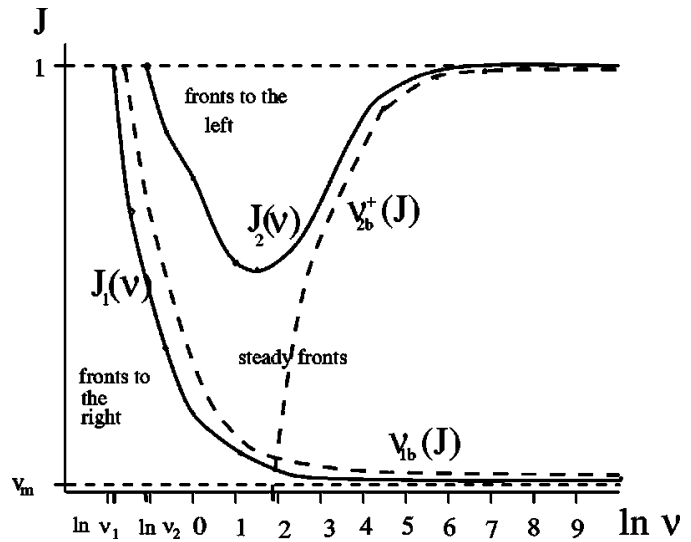

FIG. 3. Critical currents $J_{1}$ and $J_{2}$ as functions of the dimensionless doping $\nu$. Monopoles move downstream for $v_{m}<J$ $<J_{1}(\nu)$, are stationary for $J_{1}(\nu)<J<J_{2}(\nu)$, and move upstream for $J_{2}(\nu)<J<1$. Dashed lines in this figure represent the bounds $\nu_{1 b}(J)$ and $\nu_{2 b}^{+}(J)$.

velocity versus current for well doping corresponding to the 9/4 SL of Ref. [9]. Results obtained with the complete sequential tunneling current or with approximation (3) (corresponding to Fig. 4 with $\nu=3$ ) are compared. Both velocity curves are similar, and their quantitative discrepancies are irrelevant in view of the uncertainties involved in a theoretical calculation of the tunneling current (typically the offresonance current is larger than the theoretical prediction).

Once different stable monopole solutions (moving either downstream or upstream, stationary) have been identified, we raise the natural question of whether they are compatible with boundary conditions. Another series of numerical simulations was carried out to answer this. We numerically solved Eq. (13) for a current-biased finite SL $(N=40)$ with boundary conditions (10)-(12). Our results are depicted in Fig. 6 for realistic doping at the contact layers. We observe that the emitter boundary condition results in the creation of a charge accumulation layer near this contact. A charge depletion layer is formed near the collector contact as a result

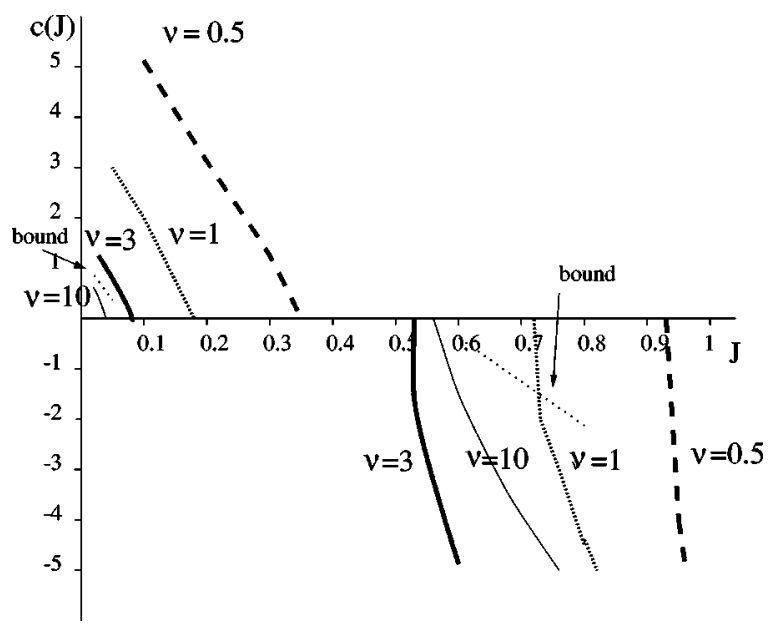

FIG. 4. Velocity of a monopole wave front as a function of $J$ for four doping values $\nu=0.5,1,3$, and 10. Monopoles with negative velocity move upstream. For doping $\nu=3$ corresponding to the $9 / 4$ SL, we have also represented bounds for the velocity as lines of thick dots.

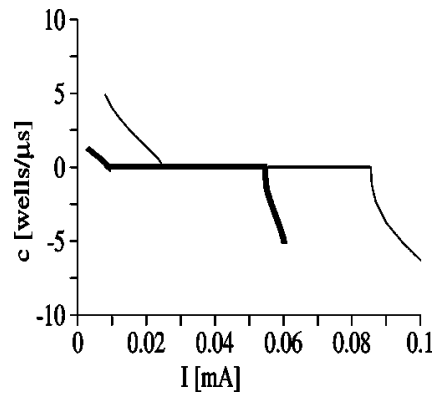

FIG. 5. Monopole velocity as a function of current for the $9 / 4$ SL. Comparison of results for the discrete drift-diffusion model [Eqs. (1) and (2)] with $\nu=3$ (thick line), and those obtained by using the exact tunneling current $e J\left(n_{i}, n_{i+1}, F_{i}\right)$ (thin line) instead of approximation (3).

of the corresponding boundary condition. Except for these layers, the existence and configuration of monopoles moving downstream, upstream or remaining stationary agrees with the previous simulations (corresponding to an infinitely long current-biased SL with a monopolelike initial condition).

\section{MATHEMATICAL ANALYSIS OF TRAVELING MONOPOLES AND STATIONARY SOLUTIONS}

In this section, we theoretically study moving or stationary monopoles on an infinitely long, current-biased SL. Our findings will confirm the picture suggested by the numerical simulations of Sec. II for any doped weakly coupled SL. Furthermore, we shall prove the stability of the different monopole solutions, and find bounds for the critical values of $\nu$ and $J_{i}$. Our results are based upon and extend ideas first proposed by Keener for discrete FitzHugh-Nagumo equations, corresponding to signal transmision in myelinated neurons [24]. Mathematically analogous problems arise in models of propagation of defects in crystals [25]. These problems have a structure

$$
\frac{d E_{i}}{d t}-d\left(E_{i+1}-2 E_{i}+E_{i-1}\right)=J-v\left(E_{i}\right),
$$

which is much simpler than Eq. (13). Here the parameter $d$ $>0$ is a constant diffusion coefficient, and $v(E)$ a "cubic", function with three branches as the electron drift velocity of Fig. 1.

For Eq. (15), there are critical values of $J, J_{1}$, and $J_{2}$, characterizing wave front behavior [24]. For $J>J_{2}(d)$, there exist wave front solutions of Eq. (15) moving upstream (to the left). For $J<J_{1}(d)$, there are wave fronts moving downstream (to the right), whereas for $J_{1}(d)<J<J_{2}(d)$, stationary fronts exist. The width of the interval $\left[J_{1}(d), J_{2}(d)\right]$ is an increasing function of $d$.

\section{A. Propagation failure and stationary solutions}

In Appendix A we state and prove a comparison principle for Eq. (13). As a consequence, if our initial field profile is monopolelike [monotone increasing with well index, and sandwiched between $E^{(1)}(J)$ and $\left.E^{(3)}(J)\right]$, so is the electric field profile for any later time $t>0$ (see Appendix A): 

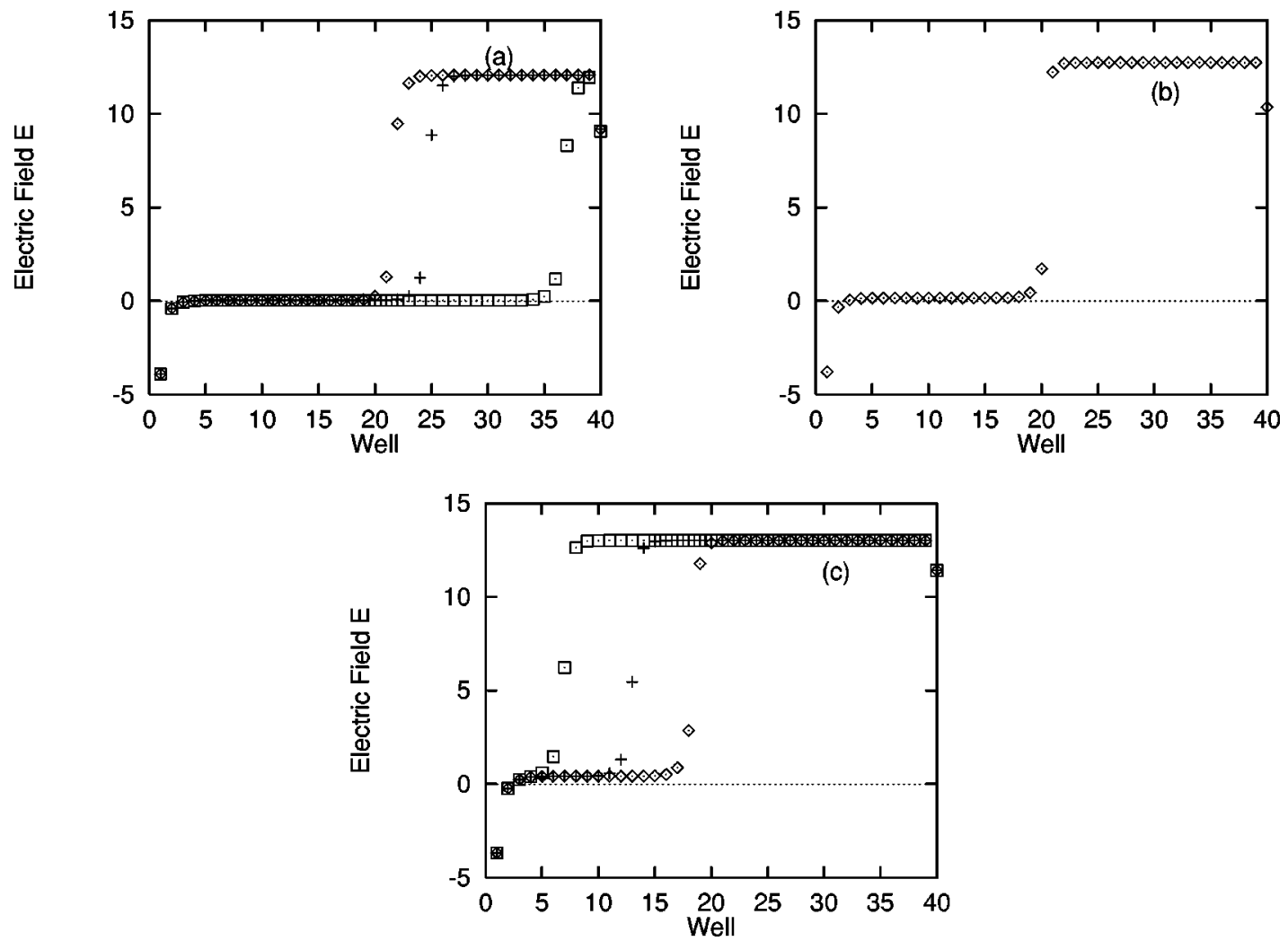

FIG. 6. Numerical simulations of the drift-diffusion model with realistic boundary conditions at the contact regions. (a) Monopole moving downstream for $J=0.023$, (b) stationary monopole for $J=0.3$, and (c) monopole moving upstream for $J=0.9$. In all cases, diamonds correspond to the profile at $t=0$, and squares to the profile at the largest positive time.

$\left\{E_{i}(0)\right\}$ increasing with $i$

$$
\Rightarrow E^{(1)}(J)<E_{i}(t)<E_{i+1}(t)<E^{(3)}(J), \quad \forall i, \quad t>0 .
$$

We now obtain sufficient conditions for an initial monopole not to propagate upstream or downstream. Under these conditions, the monopole may remain stationary or move downstream or upstream, respectively. Let us start with a condition pinning the left tail of a monopole. As $E_{i-1}<E_{i}$ and $E_{i+1}<E^{(3)}(J)$, we have

$$
\begin{aligned}
\frac{d E_{i}}{d t}= & \frac{D\left(E_{i}\right)}{\nu}\left(E_{i+1}-E_{i}\right)+\frac{D\left(E_{i}\right)+v\left(E_{i}\right)}{\nu}\left(E_{i-1}-E_{i}\right) \\
& +J-v\left(E_{i}\right) \\
\leqslant & \frac{D\left(E_{i}\right)}{\nu}\left[E^{(3)}(J)-E_{i}\right]+J-v\left(E_{i}\right) \leqslant 0,
\end{aligned}
$$

provided there exist $a_{l}<b_{l}$ such that

$$
\frac{D(E)}{\nu}\left[E-E^{(3)}(J)\right] \geqslant J-v(E), \quad E \in\left(a_{l}, b_{l}\right),
$$

and then we choose some initial field, $E_{i}(0) \in\left(a_{l}, b_{l}\right)$. The previous inequality then implies $E_{i}(t) \in\left[E^{(1)}(J), b_{l}\right]$ for all $t>0$. This in turn forbids a monopole to move upstream (to the left). We say that condition (16) pins the left tail of the monopole. Whether such $\left(a_{l}, b_{l}\right)$ exist depends on the parameters $\nu$ and $J$; see Fig. 7 .
Let us now pin the right tail of a monopole. As $E_{i+1}$ $>E_{i}$ and $E_{i-1}>E^{(1)}(J)$, we have

$$
\begin{aligned}
\frac{d E_{i}}{d t}= & \frac{D\left(E_{i}\right)}{\nu}\left(E_{i+1}-E_{i}\right)+\frac{D\left(E_{i}\right)+v\left(E_{i}\right)}{\nu}\left(E_{i-1}-E_{i}\right) \\
& +J-v\left(E_{i}\right) \\
\geqslant & \frac{D\left(E_{i}\right)+v\left(E_{i}\right)}{\nu}\left[E^{(1)}(J)-E_{i}\right]+J-v\left(E_{i}\right) \geqslant 0,
\end{aligned}
$$

provided there exist $a_{r}<b_{r}$ such that

$$
\begin{gathered}
\frac{D(E)+v(E)}{\nu}\left[E-E^{(1)}(J)\right] \leqslant J-v(E), \\
E \in\left(a_{r}, b_{r}\right),
\end{gathered}
$$

and we choose some initial field $E_{i}(0) \in\left(a_{r}, b_{r}\right)$. The previous inequality then implies $E_{i}(t) \in\left[a_{r}, E^{(3)}(J)\right]$ for all $t$ $>0$. A monopole cannot then move downstream (to the right), and we say that its right tail is pinned. Figure 8 illustrates our arguments: for fields larger than $a_{r}$, the $E_{i}$ 's tend to increase above $a_{r}$ toward $E^{(3)}(J)$. Then the monopole cannot move downstream. For $E_{i}<b_{l}$, the fields tend to $E^{(1)}(J)$, and the monopole cannot move upstream. As before, the existence of $\left(a_{r}, b_{r}\right)$ depends on the values of $\nu$ and $J$; see Fig. 7.

Figure 7 shows the curves $J-v(E), D(E)\left(E-E^{(3)}\right) / \nu$ and $[D(E)+v(E)]\left(E-E^{(1)}\right) / \nu$ for $\nu=3$ and different values of $J$. At $J=0.08$, Fig. 7(a) shows that there is an interval 

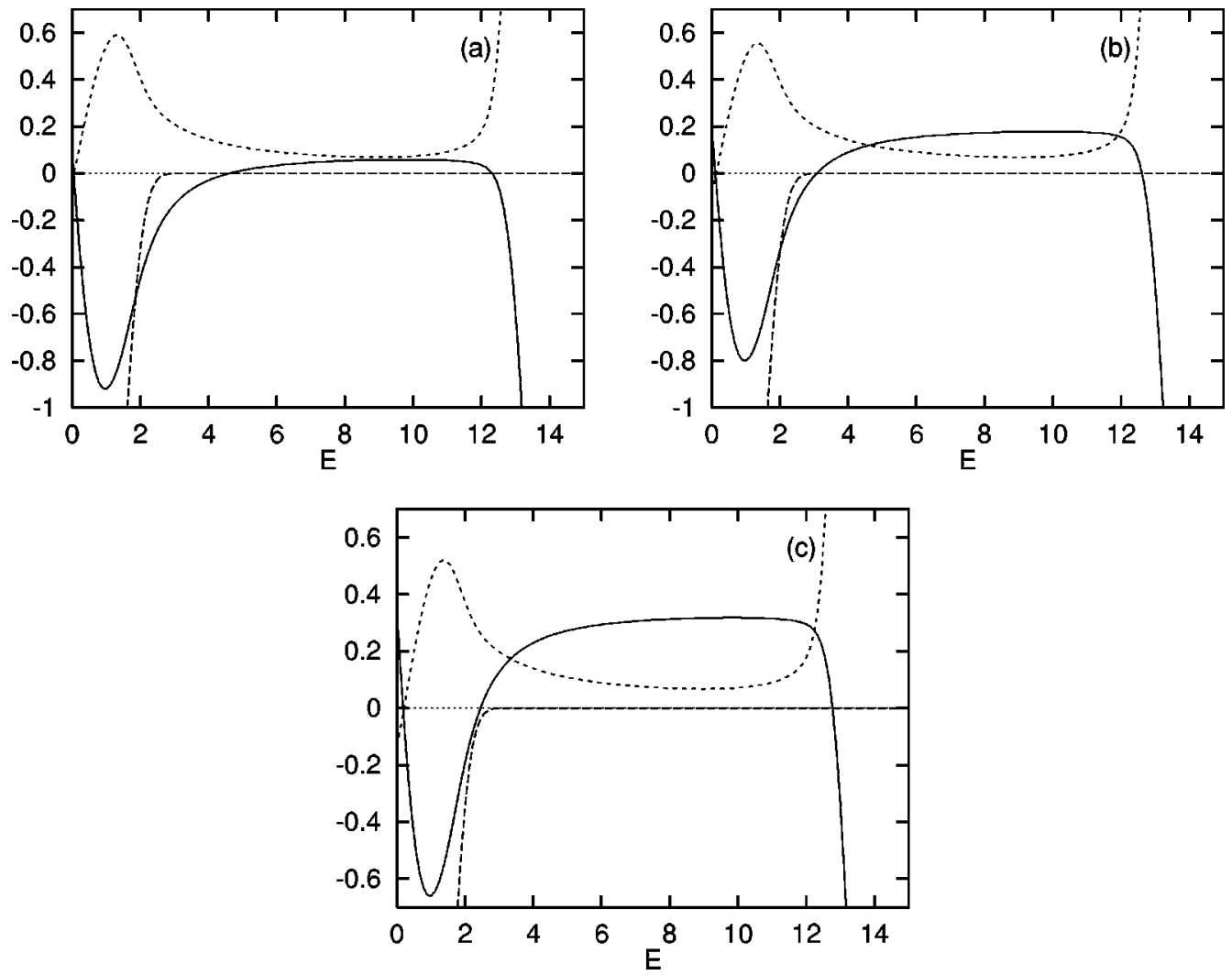

FIG. 7. (a) Functions of the electric field establishing pinning of the monopole tails for $\nu=3$ and $J=0.08$. Solid line: $J-v(E)$; dashed line: $D(E)\left[E-E^{(3)}(J)\right] / \nu$; dotted line: $[D(E)+v(E)]\left[E-E^{(1)}(J)\right] / \nu$. (b) Same plots as in (a) for $\nu=3$ and $J=0.2$. (c) Same plots as in (a) for $\nu=3$ and $J=0.34$.

$\left(a_{l}, b_{l}\right)$ as in Eq. (16), but no interval $\left(a_{r}, b_{r}\right)$ as in Eq. (17) exist. Then the left tail of a monopole is pinned, but its right tail is free. In this condition, a monopole may move downstream. Figure 7(b) shows a monopole with both its left and right tails pinned for $J=0.2$. Then our theory implies that wave front propagation fails and a monopolelike stationary solution is stable. Numerical simulations show that there are stationary solutions when $J \in[0.09,0.53]$. Finally, Fig. 7(c) shows that, if $J=0.34$, the right tail of a monopole is pinned, but not its left tail. Under these conditions a monopole may move upstream. For larger $\nu$, the estimates become sharper. For instance, when $\nu=10$, Eqs. (16) and (17) hold for $J$ $\in[0.05,0.45]$. Direct numerical simulations show that stationary solutions exist for $J \in[0.04,0.55]$. Systematic use of these criteria allows us to estimate the critical doping values $\nu_{j}$ and critical current values $J_{i}(\nu), i=1,2$ defined in Sec. II; see Appendix B. Instead of looking for $J_{1}(\nu)$ and $J_{2}(\nu)$, it is more convenient to look for their inverse functions, which we may call $\nu_{1}(J)$ and $\nu_{2}^{ \pm}(J)$. According to Fig. 3 , the inverse function of $J_{2}(\nu)$ is two-valued, and its two branches are $\nu_{2}^{-}(J)<\nu_{2}^{+}(J)$. We have found the following upper bounds $\nu_{1 b}(J)$ and $\nu_{2 b}^{+}(J)$ for $\nu_{1}(J)$ and $\nu_{2}^{+}(J)$, respectively:

$$
\begin{aligned}
\nu_{1 b}(J) & =v_{m} \frac{E_{m}-E^{(1)}(J)}{J-v_{m}}, \\
\nu_{2 b}^{+}(J) & =D(1) \frac{E^{(3)}(J)-1}{1-J} .
\end{aligned}
$$

If $\nu>\nu_{1 b}(J)$, the right tail of the monopole is pinned, whereas the left tail of the monopole is pinned if $\nu$ $>\nu_{2 b}^{+}(J)$; see Appendix B. Notice that $\nu_{1 b}(J)$ is a decreasing function of $J$. Therefore, the critical value $\nu_{1}$ (above which there are stationary solutions) is smaller than $\nu_{1 b}(1)$, which is exactly Wacker et al.'s bound, Eq. (14). This explains why bound (14) gives surprisingly good results even for the first plateau of the SL current-voltage characteristics [despite having been obtained under the assumption $D(E)$ $\equiv 0][19,18]$. Notice that bound (19) is reasonable for large dopings and currents $J \approx 1$. See Fig. 3 for a comparison between the critical curves $J_{1}(\nu)$ and $J_{2}(\nu)$ and bounds (18) and (19).
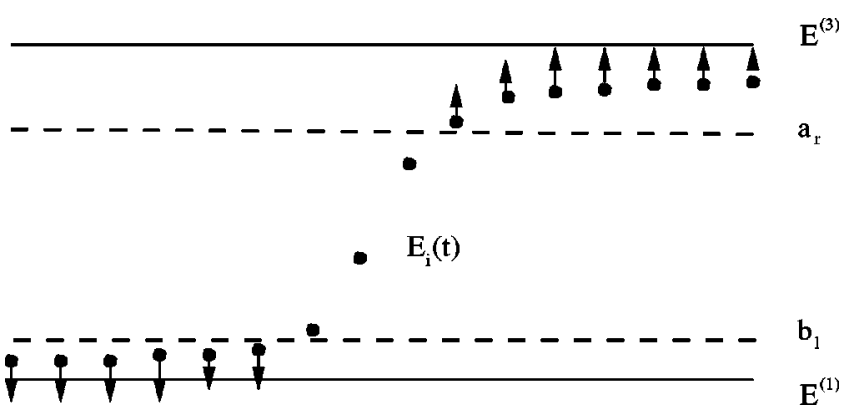

FIG. 8. A monopole field profile. The field values $b_{l}$ and $a_{r}$ are indicated by horizontal dashed lines. Vertical arrows indicate that the field at those SL periods either (i) decrease toward $E^{(1)}(J)$, and therefore the monopole left tail is pinned; or (ii) increase toward $E^{(3)}(J)$, and therefore the monopole right tail is pinned. 


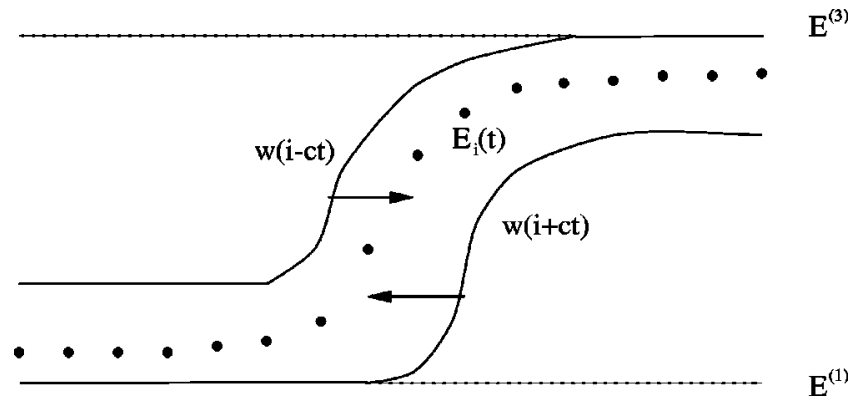

FIG. 9. A monopole field profile, subsolution and supersolution. The supersolution is always above the real values of the field, and therefore it pushes the monopole to the right. The subsolution pushes (from below) the monopole to the left.

\section{B. Propagation: traveling fronts}

Having shown that only one tail of a monopole is pinned suggests that the monopole may move in the opposite direction. Direct simulations show that this is often the case, and we will prove this now.

An upstream traveling wave solution of Eq. (13) may have the form

$$
E_{i}(t)=w(i+c t), \quad c>0 .
$$

We will look for an electric field profile $w(z), z=i+c t$, which is not an exact solution of Eq. (13), but instead satisfies

$$
\begin{aligned}
c \frac{d w}{d z}- & \frac{D[w(z)]+v[w(z)]}{\nu}[w(z-1)-w(z)] \\
& -\frac{D[w(z)]}{\nu}[w(z+1)-w(z)]+v[w(z)]-J \leqslant 0 .
\end{aligned}
$$

If this subsolution is initially below an initial field profile, i.e., $w(i)<E_{i}(0)$ for all $i$, then the comparison theorem of Appendix A guarantees that $E_{i}(t)>w(i+c t)$ for later times. As $w(i+c t)$ moves upstream, so does $E_{i}(t)$, and the electric field profile corresponds to a monopole moving upstream with velocity at least $c$. See Fig. 9: a subsolution "pushes", the monopole upstream, whereas a supersolution (defined below) "pushes" the monopole downstream.

How do we find a reasonable subsolution? An idea is to try a piecewise continuous solution which equals $E^{(1)}(J)$ for $z<z_{0}$ and a larger constant $A, A \in\left[E^{(2)}(J), E^{(3)}(J)\right]$ [and therefore $v(A)-J \leqslant 0]$, for $z>z_{1}$, with $z_{1}>z_{0}$. For $z_{0}<z$ $<z_{1}, w(z)$ is an unspecified smooth increasing function with $w\left(z_{0}\right)=E^{(1)}(J)$ and $w\left(z_{1}\right)=A$. Now we shall conveniently select the numbers $z_{0}, z_{1}, c$, and $A$, so that Eq. (21) holds. Clearly, Eq. (21) holds for $z+1<z_{0}$ and $z-1>z_{1}$. Suppose that $0<z_{1}-z_{0}<1$. Then there are five possibilities:

(1) $z<z_{0}$ and $z+1>z_{1}$. Then $w(z-1)=w(z)=E^{(1)}(J)$ and $w(z+1)=A$, which, inserted in Eq. (21) yields $-D\left(E^{(1)}\right)\left(A-E^{(1)}\right) / \nu \leqslant 0$ (obviously true).

(2) $z-1<z_{0}$ and $z>z_{1}$. Then $w(z-1)=E^{(1)}(J)$ and $w(z)=w(z+1)=A$, which inserted in Eq. (21) yields

$$
J-v(A) \geqslant \frac{D(A)+v(A)}{\nu}\left[A-E^{(1)}(J)\right] .
$$

(3) $z_{0}<z-1<z_{1}$. Then $E^{(1)}(J)<w(z-1)<A$ and $w(z)$ $=w(z+1)=A$, which yields $J-v(A) \geqslant[D(A)+v(A)][A$ $-w(z-1)] / \nu$. This inequality holds if Eq. (22) does.

(4) $z_{0}<z<z_{1}$. Then $w(z-1)=E^{(1)}(J)$ and $E^{(1)}(J)$ $<w(z)<A$ and $w(z+1)=A$. Inserting this into Eq. (21), we find

$$
\begin{aligned}
c \frac{d w}{d z} \leqslant & \frac{D[w(z)]+v[w(z)]}{\nu}\left[E^{(1)}-w(z)\right] \\
& +\frac{D[w(z)]}{\nu}[A-w(z)]+J-v[w(z)] .
\end{aligned}
$$

Let us now assume that we can select $A \in\left(E^{(2)}, E^{(3)}\right)$ such that the right hand side of this expression is positive, say

$$
\begin{gathered}
\frac{D(w)+v(w)}{\nu}\left[E^{(1)}(J)-w\right]+\frac{D(w)(A-w)}{\nu} \\
+J-v(w) \geqslant \delta>0, \quad E^{(1)}<w<A,
\end{gathered}
$$

and that we choose $c$ so that $c d w / d z<\delta$. Then Eq. (21) holds.

(5) $z_{0}<z+1<z_{1}$. Then $w(z-1)=w(z)=E^{(1)}(J)$ and $E^{(1)}(J)<w(z+1)<A$, which inserted into Eq. (21), yields $-D\left(E^{(1)}\right)\left[w(z+1)-E^{(1)}\right] / \nu \leqslant 0$ (obviously true).

Summarizing the previous arguments, provided Eqs. (22) and (23) hold, $w(z)$ is a subsolution obeying Eq. (21). The parameter $A$ can be found graphically. First of all, we depict the functions $J-v(E)$ and $f_{1}(E ; J) \equiv[D(E)+v(E)][E$ $\left.-E^{(1)}(J)\right] / \nu$. Possible values of $A$ are those $E$ for which $J$ $-v(E) \geqslant f_{1}(E ; J)$. For such a value of $A$, we may plot the left side of Eq. (23):

$$
\begin{aligned}
f_{2}(E ; J, A) \equiv & \frac{D(E)+v(E)}{\nu}\left[E^{(1)}(J)-E\right] \\
& +\frac{D(E)(A-E)}{\nu}+J-v(E) .
\end{aligned}
$$

If $f_{2}(E ; J, A)>0$ for $E \in\left[E^{(1)}(J), A\right]$, then the selected value of $A$ allows us to construct the sought subsolution. See Fig. 10 for a practical realization of this graphical construction.

We have proved rigorously that monopoles may move upstream under favorable circumstances. Our proof, using subsolutions, may yield a very practical additional bonus: an upper bound $c^{*}$ for the velocity of the monopole. Let us choose $\delta(J, A)=\min _{E^{(1)}<E<A} f_{2}(E ; J, A), z_{1}-z_{0}=1$, and $w(z)$ $=\left[A-E^{(1)}(J)\right]\left(z-z_{0}\right)$ for $z_{0}<z<z_{1}$. Then $c^{*}=\delta(J, A) /$ $\left[A-E^{(1)}(J)\right]$. In Fig. 4, $-c^{*}$ is represented by a line of thick dots for doping $\nu=3$ corresponding to the 9/4 SL.

In a similar vein, we can construct supersolutions which push the monopole field profile to the right; see Fig. 9. Now we start from a monopole profile moving downstream:

$$
E_{i}(t)=w(i-c t), \quad c>0
$$

The electric field profile $w(z), z=i-c t$ should satisfy 


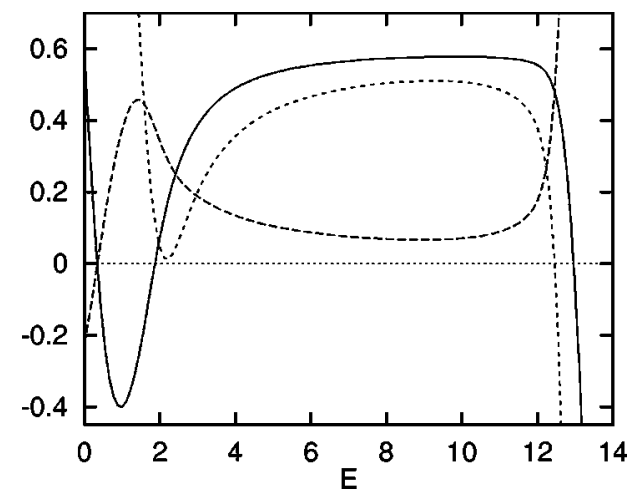

FIG. 10. Curves determining the subsolution for $\nu=3, J=0.6$, and $A=12$. Solid line: $J-v(E)$; dashed line: $f_{1}(E ; J)$; dotted line: $f_{2}(E ; J, A)$.

$$
\begin{aligned}
c \frac{d w}{d z} & +\frac{D[w(z)]+v[w(z)]}{\nu}[w(z-1)-w(z)] \\
& +\frac{D[w(z)]}{\nu}[w(z+1)-w(z)]+J-v[w(z)] \leqslant 0 .
\end{aligned}
$$

We seek a piecewise continuous supersolution which equals a constant, $A, A \in\left[E^{(1)}(J), E^{(2)}(J)\right]$, for $z<z_{0}$, and $w(z)$ $=E^{(3)}(J)$ for $z>z_{1}$, with $z_{1}>z_{0}$. For $z_{0}<z<z_{1}, w(z)$ is an unspecified smooth increasing function with $w\left(z_{0}\right)=A$, and $w\left(z_{1}\right)=E^{(3)}(J)$. As for subsolutions, we now select conveniently the numbers $z_{0}, z_{1}, c$ and $A$ so that Eq. (26) holds. Clearly, Eq. (26) holds for $z+1<z_{0}$ and for $z-1>z_{1}$. Suppose that $0<z_{1}-z_{0}<1$. An analysis of the remaining five possibilities yields the following criteria to hold for $w(i$ $-c t$ ) to be a supersolution:

$$
\begin{gathered}
J-v(A) \leqslant-\frac{D(A)}{\nu}\left[E^{(3)}(J)-A\right] \equiv f_{3}(A ; J) \\
f_{4}(w ; J, A) \equiv-\frac{D(w)+v(w)}{\nu}(w-A)+\frac{D(w)}{\nu}\left[E^{(3)}(J)-w\right] \\
+J-v(w) \leqslant-\delta
\end{gathered}
$$

for

$$
\begin{gathered}
A \leqslant w \leqslant E^{(3)}(J), \\
c \frac{d w}{d z} \leqslant \delta .
\end{gathered}
$$

Provided such $w(i-c t)$ is found, solutions $E_{i}(t)$ of Eq. (13) with $E_{i}(0)<w(i+\tau)$ will satisfy $E_{i}(t)<w(i-c t+\tau)$, and propagate to the right with speed larger than $c . \tau$ is a constant which can be conveniently chosen to keep the monopole profile below the supersolution. Figure 11 illustrates the graphical construction of the supersolution by checking that Eqs. (27) and (28) hold for particular values of $J$ and $\nu$.

As in the subsolution case, an upper bound $c^{*}$ for the monopole velocity $c$ is estimated by choosing $-\delta(J, A)$

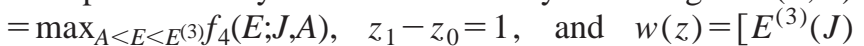
$-A]\left(z-z_{0}\right)$ for $z_{0}<z<z_{1}$. Then $c^{*}=\delta(J, A) /\left[E^{(3)}(J)\right.$

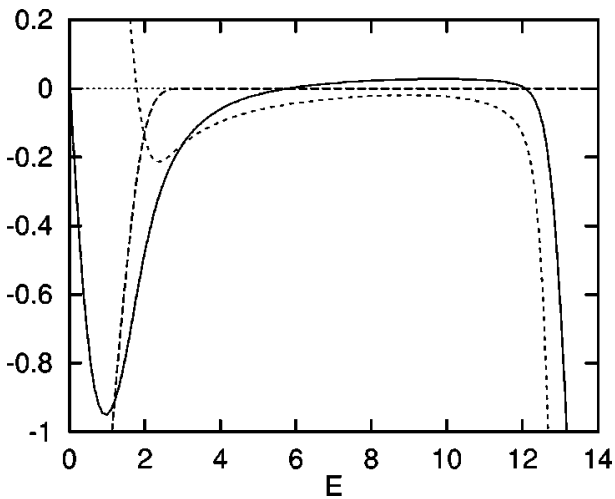

FIG. 11. Curves determining the supersolution for $\nu=3, J$ $=0.05$, and $A=3$. Solid line: $J-v(E)$; dashed line: $f_{3}(E ; J)$; dotted line: $f_{4}(E ; J, A)$.

$-A$ ]. In Fig. $4, c^{*}$ is represented by a line of thick dots for doping $\nu=3$ corresponding to the 9/4 SL.

We can now summarize the results obtained from sub and supersolutions; see Figs. 10 and 11. We find reasonably good upper bounds for the absolute value of the monopole velocity. Furthermore, for $\nu=3$, conditions (22) and (23) hold for $J=0.6$ and $A=12$, whereas conditions (27) and (28) hold for $J=0.05$ and $A=3$. Therefore, monopoles move downstream for $J \leqslant 0.05$, and they move upstream for $J \geqslant 0.6$. Direct numerical simulations show that (i) the estimate $J_{1}=0.05$ for the first critical current can be improved to $J_{1}=0.08$; and (ii) the estimate $J_{2}=0.6$ for the second critical current can be improved to $J_{2}=0.54$.

\section{CONCLUSIONS AND FINAL COMMENTS}

We have presented a theory of monopoles moving downstream or upstream on an infinitely long doped, currentbiased superlattice when the fields are on the first plateau of the current-voltage characteristic. This theory has been corroborated with numerical evidence, which sharpens our results. Furthermore, we have simulated a 40-well 9-nmGaAs/ 4-nmAlAs SL [9] under doping and contact conditions similar to experimental ones [21], but under constant current bias conditions. This situation is different from the usual case of voltage bias conditions. We have obtained that it is possible to observe monopole wave fronts moving upstream when the current is kept at large enough levels. Together with our theoretical bounds for critical currents and dopings, this numerical prediction could be used to set up an experiment to observe this striking phenomenon. For this purpose, we would need an initial condition corresponding to a monopole separating two electric field domains at high enough current. In an ideal world, this situation could be obtained by first fixing a low dc voltage for the $9 / 4$ sample at a value near the top of one of the first branches of the current-voltage characteristics. Then we could switch from voltage to current bias conditions. The outcome would be a monopole moving upstream until the emitter region is reached. Presumably an idea of the field distribution corresponding to this situation could be obtained by time-resolved photoluminescence measurements [8].

There are technical problems that must be overcome if one wants to observe these features in real experiments: 
when we switch, there will always be a Faraday-like inductive pulse which will probably perturb the state of the system in an uncontrolled way. There are other possible biases we could think of. Under dc voltage bias, monopoles moving upstream are probably created for a short time during relocation experiment [30]. In these experiments, one has a doped SL with a current-voltage characteristics corresponding to multiple stationary monopole solution branches. Voltage is set at a particular value near the end of a branch, so that the field profile is that of a monopole layer connecting a low to a high field domain. Let the monopole layer be located at well $i$ (counted from the emitter contact). Then the voltage is suddenly and appropriately increased. After a certain time, the field profile settles to a new situation corresponding to a monopole layer centered at well $i-1$ [30]. This could be an indication of a monopole moving upstream, albeit for a short time. To increase this time, we could try to set a hybrid bias (between current and voltage bias) by including a finite series resistance in our external circuit. Additional theoretical and numerical work is needed to explore these possibilities.

\section{ACKNOWLEDGMENTS}

We thank A. Amann, G. Platero, and D. Sánchez for fruitful discussions and collaboration on the discrete driftdiffusion model. L.L.B. thanks S.W. Teitsworth for a critical reading of the manuscript and helpful comments. This work was supported by the Spanish DGES through Grant No. PB98-0142-C04-01, and by the European Union TMR Contract Nos. ERB FMRX-CT96-0033 and ERB FMRX-CT970157 .

\section{APPENDIX A: COMPARISON PRINCIPLE}

The main theorem which we use to prove our results in Sec. III is the following comparison principle:

Theorem A.1: Let $U_{i}(t)$ and $L_{i}(t), i \in Z$, be differentiable sequences such that

$$
\begin{aligned}
\frac{d U_{i}}{d t} & -d_{1}\left(U_{i}\right)\left[U_{i+1}-U_{i}\right]-d_{2}\left(U_{i}\right)\left[U_{i-1}-U_{i}\right]-f\left(U_{i}\right) \\
& \geqslant \frac{d L_{i}}{d t}-d_{1}\left(L_{i}\right)\left[L_{i+1}-L_{i}\right]-d_{2}\left(L_{i}\right)\left[L_{i-1}-L_{i}\right]-f\left(L_{i}\right),
\end{aligned}
$$

$$
U_{i}(0)>L_{i}(0)
$$

where $f, d_{1}>0$ and $d_{2}>0$ are Lipschitz continuous functions. Then,

$$
U_{i}(t)>L_{i}(t), \quad t>0, i \in Z
$$

In our discrete drift-diffusion model,

$$
\begin{gathered}
d_{1}(E)=\frac{D(E)}{\nu}, \quad d_{2}(E)=\frac{D(E)+v(E)}{\nu}, \\
f(E)=J-v(E) .
\end{gathered}
$$

Proof: The proof is by contradiction. Set $W_{i}(t)=U_{i}(t)$ $-L_{i}(t)$. At $t=0, W_{i}(0)>0$ for all $i$. Let us assume that $W_{i}$ changes sign after a certain minimum time $t_{1}>0$, at some value of $i, i=k$. Thus $W_{k}\left(t_{1}\right)=0$ and $d W_{k} / d t \leqslant 0$, as $t$ $\rightarrow t_{1}$. We shall show that this is contradictory. At $t=t_{1}$, there must be an index $m$ (equal or different from $k$ ) such that $W_{m}\left(t_{1}\right)=0$, while its next neighbor $W_{m+j}\left(t_{1}\right)>0(j$ is either 1 or -1$)$, and $W_{i}\left(t_{1}\right)=0$ for all indices between $k$ and $m$. For otherwise $W_{k}$ should be identically 0 for all $k$. Equation (A1) implies

$$
\begin{aligned}
\frac{d W_{m}}{d t}\left(t_{1}\right) & \geqslant d_{1}\left(U_{m}\left(t_{1}\right)\right) W_{m+1}\left(t_{1}\right)+d_{2}\left(U_{m}\left(t_{1}\right)\right) W_{m-1}\left(t_{1}\right) \\
& >0
\end{aligned}
$$

This contradicts the fact that $d W_{m} / d t$ should have been nonpositive as $t \rightarrow t_{1}$, for $W_{m}\left(t_{1}\right)$ to have become zero in the first place.

Corollary A.1: Any solution $E_{i}(t)$ of Eq. (13) with initial data $E_{i}(0) \in\left[E^{(1)}(J), E^{(3)}(J)\right] \quad$ satisfies $E_{i}(t)$ $\in\left[E^{(1)}(J), E^{(3)}(J)\right]$ for $t>0$.

Proof: Apply theorem A.1 first with $L_{i}=E^{(1)}(J)$ and $U_{i}$ $=E_{i}$, then with $L_{i}=E_{i}$ and $U_{i}=E^{(3)}(J)$.

Corollary A.2: If $E_{i}(0)$ is monotone increasing, that is, $E_{i}(0)<E_{i+1}(0)$, then, $E_{i}(t)$ is also monotone increasing, i.e., $E_{i}(t)<E_{i+1}(t)$ for $t>0$.

Proof: Apply theorem A.1 with $L_{i}=E_{i}(t)$ and $U_{i}$ $=E_{i+1}(t)$.

Remark: Strict inequalities in these theorems can be replaced by inequalities, and the corresponding statements still hold. However, the proofs become rather more technical and involved.

\section{APPENDIX B: BOUNDS FOR CRITICAL DOPING VALUES}

We want to estimate the curves $\nu_{1 b}(J)$ and $\nu_{2 b}^{ \pm}(J)$ defined in Sec. III. To estimate $\nu_{2 b}^{+}(J)$, assume that $J \rightarrow 1-$ and $\nu$ is large. The left tail of a monopole is pinned if Eq. (16) holds. For large currents, Eq. (16) certainly holds if the curve corresponding to the left side of the inequality is above that of the right hand side, for $E=1$ [this is possible because $D(E)$ decreases rapidly to zero as the field increases]. Setting $E$ $=1$ in Eq. (16), we obtain

$$
\frac{D(1)\left[1-E^{(3)}(J)\right]}{\nu}>J-1 .
$$

In turn, this implies $\nu>\nu_{2 b}^{+}(J)$, defined in Eq. (19). This argument fails for the small values of $J$ used to draw Fig. 7. We believe that quite different reasoning is needed to estimate $\nu_{2 b}^{-}(J)$.

The same argument yields our estimate $\nu_{1 b}(J)$ of Eq (18). For Eq. (17) to hold, the curve corresponding to the left side of the inequality should be below that of the right hand side for $E=E_{m}$. As $D\left(E_{m}\right) \approx 0$, we obtain

$$
\frac{v\left(E_{m}\right)}{\nu}\left[E_{m}-E^{(1)}(J)\right]<J-v\left(E_{m}\right),
$$

which yields Eq. (18). Figure 3 shows that bound (18) is reasonably good for all eligible values of $\nu$ and $J$. 
[1] L. Esaki and R. Tsu, IBM J. Res. Dev. 14, 61 (1970).

[2] M. Büttiker and H. Thomas, Phys. Rev. Lett. 38, 78 (1977); Z. Phys. B: Condens. Matter 34, 301 (1979); X. L. Lei, N. J. M. Horing, and H. L. Cui, Phys. Rev. Lett. 66, 3277 (1991); J. C. Cao and X.L. Lei, Phys. Rev. B 60, 1871 (1999).

[3] A. Sibille, J. F. Palmier, F. Mollot, H. Wang, and J. C. Esnault, Phys. Rev. B 39, 6272 (1989).

[4] R. Tsu and G. H. Döhler, Phys. Rev. B 12, 680 (1975); S. Rott, N. Linder, and G. H. Döhler, Superlattices Microstruct. 21, 569 (1997).

[5] J. B. Gunn, Solid State Commun. 1, 88 (1963).

[6] L. Esaki and L. L. Chang, Phys. Rev. Lett. 33, 495 (1974).

[7] Y. Kawamura, K. Wakita, H. Asahi, and K. Kurumada, Jpn. J. Appl. Phys. 25, L928 (1986); K. K. Choi, B. F. Levine, R. J. Malik, J. Walker, and C. G. Bethea, Phys. Rev. B 35, 4172 (1987); M. Helm, P. England, E. Colas, F. DeRosa, and S.J. Allen, Jr., Phys. Rev. Lett. 63, 74 (1989); H. T. Grahn, R. J. Haug, W. Müller, and K. Ploog, ibid. 67, 1618 (1991); J. Kastrup, H. T. Grahn, K. Ploog, F. Prengel, A. Wacker, and E. Schöll, Appl. Phys. Lett. 65, 1808 (1994); S. H. Kwok, H. T. Grahn, M. Ramsteiner, K. Ploog, F. Prengel, A. Wacker, E. Schöll, S. Murugkar, and R. Merlin, Phys. Rev. B 51, 9943 (1995); Y.A. Mityagin, V.N. Murzin, Y.A. Efimov, and G.K. Rasulova, Appl. Phys. Lett. 70, 3008 (1997).

[8] J. Kastrup, R. Klann, H. T. Grahn, K. Ploog, L. L. Bonilla, J. Galán, M. Kindelan, M. Moscoso, and R. Merlin, Phys. Rev. B 52, 13761 (1995).

[9] J. Kastrup, R. Hey, K. Ploog, H. T. Grahn, L. L. Bonilla, M. Kindelan, M. Moscoso, A. Wacker, and J. Galán, Phys. Rev. B 55, 2476 (1997).

[10] D. Sánchez, M. Moscoso, L. L. Bonilla, G. Platero, and R. Aguado, Phys. Rev. B 60, 4489 (1999).

[11] O. M. Bulashenko and L. L. Bonilla, Phys. Rev. B 52, 7849 (1995); O. M. Bulashenko, M. J. García, and L. L. Bonilla, ibid. 53, 10008 (1996).

[12] Y. Zhang, J. Kastrup, R. Klann, K. Ploog, and H. T. Grahn, Phys. Rev. Lett. 77, 3001 (1996); K. J. Luo, H. T. Grahn, K. H. Ploog, and L. L. Bonilla, ibid. 81, 1290 (1998).

[13] B. Sun, J. Wang, W. Ge, Y. Wang, D. Jiang, H. Zu, H. Wang, Y. Deng, and S. Feng, Phys. Rev. B 60, 8866 (1999).
[14] N. Ohtani, N. Egami, H. T. Grahn, K. H. Ploog, and L. L. Bonilla, Phys. Rev. B 58, R7528 (1998).

[15] F. Prengel, A. Wacker, and E. Schöll, Phys. Rev. B 50, 1705 (1994); 52, 11 518(E) (1995).

[16] L. L. Bonilla, J. Galán, J. A. Cuesta, F. C. Martínez, and J. M. Molera, Phys. Rev. B 50, 8644 (1994).

[17] L. L. Bonilla, in Nonlinear Dynamics and Pattern Formation in Semiconductors and Devices, edited by F.-J. Niedernostheide (Springer, Berlin, 1995), pp. 1-20; L. L. Bonilla, M. Kindelan, M. Moscoso, and S. Venakides, SIAM (Soc. Ind. Appl. Math.) J. Appl. Math. 57, 1588 (1997).

[18] A. Wacker, in Theory and Transport Properties of Semiconductor Nanostructures, edited by E. Schöll (Chapman and Hall, London, 1998), Chap. 10.

[19] A. Wacker, M. Moscoso, M. Kindelan, and L. L. Bonilla, Phys. Rev. B 55, 2466 (1997).

[20] M. Patra, G. Schwarz, and E. Schöll, Phys. Rev. B 57, 1824 (1998).

[21] L. L. Bonilla, G. Platero, and D. Sánchez, SISSA e-print, cond-mat/9909449.

[22] D. G. Aronson and H. F. Weinberger, SIAM Rev. 20, 245 (1978); Adv. Math. 30, 33 (1978); Lect. Notes Math. 446, 5 (1975).

[23] J. P. Keener and J. Sneyd, Mathematical Physiology (Springer, New York, 1998), Chap. 9.

[24] J. P. Keener, SIAM (Soc. Ind. Appl. Math.) J. Appl. Math. 47, 556 (1987).

[25] F. R. N. Nabarro, Theory of Crystal Dislocations (Oxford University Press, Oxford, 1967).

[26] A. Carpio, S. J. Chapman, S. Hastings, and J.B. Macleod, Eur. J. Appl. Math. (to be published).

[27] G. Grüner and A. Zettl, Phys. Rep. 119, 117 (1985); G. Grüner, Rev. Mod. Phys. 60, 1129 (1988); A. A. Middleton, Phys. Rev. Lett. 68, 670 (1992).

[28] T. Kontorova and Ya.I. Frenkel, Zh. Éksp. Teor. Fiz. 8, 1340 (1938) [Sov. Phys. JETP 8, 1389 (1938)]; J. Frenkel and T. Kontorova, Phys. Z. Sowjetunion 13, 1 (1938); Fiz. Zh. 1, 137 (1939).

[29] A. Wacker and A.-P. Jauho, Phys. Rev. Lett. 80, 369 (1998).

[30] K. J. Luo, H. T. Grahn, and K. H. Ploog, Phys. Rev. B 57, R6838 (1997). 